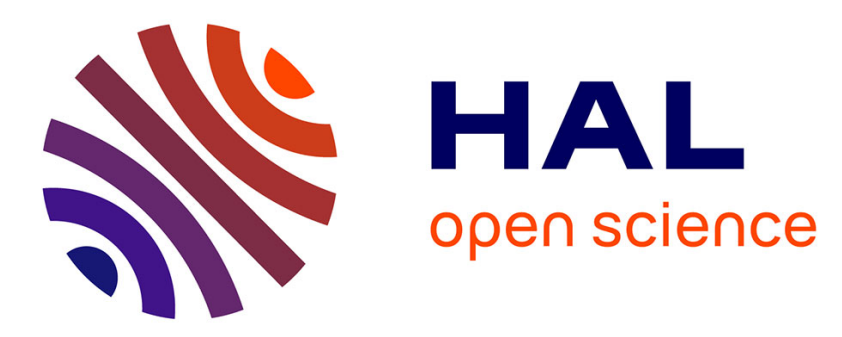

\title{
L'école maternelle: un moment clef dans la scolarisation des élèves sourds ou malentendants
}

\author{
Anne Vanbrugghe
}

\section{To cite this version:}

Anne Vanbrugghe. L'école maternelle: un moment clef dans la scolarisation des élèves sourds ou malentendants. La nouvelle revue de l'adaptation et de la scolarisation, 2009, 2 (46), pp.137-147. hal-01663088

\section{HAL Id: hal-01663088 \\ https://hal-inshea.archives-ouvertes.fr/hal-01663088}

Submitted on 13 Dec 2017

HAL is a multi-disciplinary open access archive for the deposit and dissemination of scientific research documents, whether they are published or not. The documents may come from teaching and research institutions in France or abroad, or from public or private research centers.
L'archive ouverte pluridisciplinaire HAL, est destinée au dépôt et à la diffusion de documents scientifiques de niveau recherche, publiés ou non, émanant des établissements d'enseignement et de recherche français ou étrangers, des laboratoires publics ou privés. 


\title{
L'école maternelle: un moment clef dans la scolarisation des élèves sourds ou malentendants
}

\author{
Anne VANBRUGGHE \\ Formatrice à I'INS HEA
}

Résumé: Cet article tente de faire le point sur les enjeux de la scolarisation des élèves sourds ou malentendants à l'école maternelle. II souligne la nécessité pour l'élève sourd de disposer précocement d'une langue véhiculaire des apprentissages, qu'elle soit vocale ou gestuelle. L'acquisition de la Langue des signes française apparaît ici comme un vecteur d'intégration sociale, par le fait qu'elle permet l'accès à des connaissances et à des valeurs socioculturelles partagées par tous. L'élève sourd devient alors citoyen à part entière, capable de comprendre, réfléchir et décider par lui-même. En outre, la LSF constitue un objet d'apprentissage linguistique et culturel commun qui place l'élève sourd dans une position d'échange équitable et modifie positivement le regard porté sur lui. L'article insiste également sur l'importance de la découverte et l'expérimentation du monde sonore en relation avec le quotidien, I'univers musical ou la parole vocale, dans un cadre sécurisant et adapté. II appelle de ses vœux des pratiques pédagogiques différenciées, à partir d'expériences positives et plaisantes n'allant jamais au-delà des possibilités psycho-physiologiques de l'élève sourd. L'articulation entre les actions pédagogiques et rééducatives, parfois encore confondues, ne sera effective que lorsque les champs d'intervention des enseignants et des orthophonistes se trouveraient, discutés, clarifiés et assumés pleinement.

Mots-clés: Bilinguisme - Déficient auditif - École maternelle - Scolarisation - Surdité.

\section{Nursery school: a key moment in the schooling of deaf or hearing-impaired pupils}

Summary: This article seeks to provide an update on the challenges of schooling deaf or hearing-impaired pupils in the nursery school. It stresses the need for the deaf pupils to have, at an early stage, a common language for learning, be it vocal or gestural. The acquisition of the French Sign Language appears in this context to be a vector of social integration as it opens up access to knowledge and socio-cultural values shared by all. The deaf pupil thus becomes a full-fledged citizen, able to understand, think, and decide for himself. Moreover, French Sign Language constitutes an object of linguistic learning and common culture which places the deaf pupil in a position of equal exchange, and positively changes the way that others perceive him. The article also stresses the importance of discovery and experimentation of the world of sounds in everyday life, the world of music or the spoken word, in a safe and adapted framework. The co-ordination between pedagogical activities and re-educational activities, which are still sometimes confused with one another, will become real only when the fields of action of teachers and speech therapists are discussed, clarified, and fully accepted.

Key words: Bilingualism - Deafness - Hearing impaired - Nursery School - Schooling. 
"Tu es grand maintenant, demain tu vas à l'école ": cette phrase, prononcée par des milliers de parents, résonne différemment selon les enfants. Alors que certains ne feront que déceler, en cette veille de première rentrée, une solennité mêlée de stress, d'autres en savent déjà long et en parlent depuis plusieurs mois. L'élève sourd l'aura-t-il même entendu cette phrase, comme toutes les autres qui l'ont précédé? À moins, peut-être, qu'elle lui ait été dite en langue des signes... Quelles expériences, quelles représentations, quels mots ou quels signes lui permettront de se projeter, de parler de ce qu'il voit, de ce qu'il vit, de ce qu'il veut et de ce qu'il pense, et de comprendre ce si grand jour qui l'attend et tout ceux qui suivront? L'entendant de deux ou trois ans, me direz-vous, ne comprend pas bien non plus ce qui l'attend en passant la porte de la maternelle. D'aucuns n'en auront toujours pas tout compris en sortant. Une fois admis qu'il y a parfois plus de différences entre deux entendants qu'entre un entendant et un sourd, comparons ce qui est comparable et voyons dans quelle situation particulière se trouvent les élèves sourds face aux situations qu'ils vivront à l'école maternelle.

Au-delà de la décentration qu'imposent les questions liées à la surdité chez l'entendant, elles font apparaître une réalité hétérogène. Vouloir une réponse simple et unique relève de l'utopie ou de la prétention. Aussi, convient-il de préciser que les positions ou situations évoquées correspondent plus à des stéréotypes qu'à une multitude de variantes. Rappelons en effet, qu'entre deux conjonctures opposées le cas de l'enfant sourd profond signeur précoce, de famille socio-culturellement favorisée et celui de l'enfant malentendant, "parlant tardif " de famille défavorisée, existe une multitude de situations diverses dont il est difficile de rendre compte exhaustivement.

Déterminer ce qu'il faut pour scolariser un enfant sourd, dans des conditions au moins aussi équitables que les autres, suppose de croiser plusieurs faisceaux d'observations et d'éclairer alternativement l'enfant, les attentes de l'école, les situations auxquelles il va être confronté. Les obstacles ne manqueront pas de jalonner son parcours, ils doivent être pensés au regard des moyens dont il dispose pour les surmonter : soit personnellement, soit grâce à des aides mises à sa disposition. Nous tenterons de dessiner les contours de cet enfant sourd ou malentendant, non pas simplement en référence à la norme entendante qui conduirait à le définir essentiellement comme non entendant, mais davantage à travers ses capacités singulières, ses stratégies, ses comportements, afin de déterminer ce sur quoi l'enseignant peut compter positivement. Nous verrons alors comment un regard porté initialement sur l'oreille et la bouche, lieux de toutes les difficultés, pourra se déplacer progressivement vers l'œil et la main, creusets de toutes les potentialités, parmi lesquelles celle de générer du signe, d'exprimer une pensée, bel et bien intacte chez l'enfant sourd. La capacité de langage vocal et gestuel inscrite génétiquement chez l'humain, se développant dans l'interaction avec son environnement, nous insisterons sur l'importance de laisser émerger dès le plus jeune âge chez l'enfant sourd ou malentendant sa gestualité naturelle, sans craindre de lui voir perdre ses capacités éventuelles à parler vocalement.

Nous mettrons en évidence la capacité des projets bilingues (Langue des signes/ français) à mobiliser activement non seulement l'élève sourd mais aussi ses camarades entendants, la langue des signes et la culture sourde devenant objet 
d'échange, de partage équitable, d'apprentissage pour tous. Enfin, nous évoquerons la nécessité dès l'école maternelle de faire découvrir le monde sonore à l'élève sourd, nous montrerons comment les perspectives rééducatives et pédagogiques se complètent sans se confondre.

\section{ค) DU STIGMATE AU DÉNI DE HANDICAP: SE FRAYER UN CHEMIN ENTRE DEUX IMPASSES PÉDAGOGIQUES}

Si le rapport à l'autre se nourrit en partie de l'idée préalable qu'on se fait de lui, l'enseignant devra interroger ses représentations de la surdité, comme autant de préjugés qui vont influencer précocement et durablement la relation pédagogique à l'élève sourd et la conception des dispositifs d'apprentissages qui lui sont dédiés. Deux grands pôles idéologiques situés aux extrêmes d'un continuum aboutissent, selon nous, à deux impasses pédagogiques, entre lesquelles il faudra bien se frayer un chemin. Comment ne pas faire de la surdité un stigmate faisant immanquablement de la personne sourde un invalide? A contrario, comment ne jamais perdre de vue que l'on a devant soi quelqu'un qui n'entend pas, puisque sa surdité ne se rappelle pas à nous à chaque instant? La corde est raide et le pédagogue, à la fois funambule et géomètre selon Diatkine, aura toutes les peines à maintenir l'équilibre et à ne pas basculer de l'un ou l'autre côtés.

Le premier écueil consiste donc à considérer l'enfant sourd non plus comme une personne ordinaire, faite de particularités formant un tout singulier, mais comme un être a priori singulier et avant tout défini par sa surdité. L'élève sourd est-il un élève comme les autres ou un élève handicapé ? En d'autres termes et à l'inverse, les autres élèves ne sont-ils pas eux aussi des élèves différemment handicapés? Certains se trouveront parfois plus en difficulté que l'élève sourd pour nager, dessiner ou même communiquer. II ne s'agit pas ici d'un simple jeu de mots où la sémantique finirait par y perdre son latin.

L'élève dit "en situation de handicap ", quelle qu'en soit l'origine, rappelle au pédagogue à quel point une pensée trop centrée sur le manque, la difficulté ou la déficience, ne peut conduire qu'à une voie sans issue.

En effet, pour l'expert, l'évidente maladresse de l'apprenti masque souvent ses compétences, de même, aux yeux de la normalité, le handicap apparaît comme un gouffre béant à combler, creusé comme autant d'irrégularités à corriger et l'on peine à voir les capacités poindre derrière l'interminable liste des incapacités. Le pédagogue ne peut pas ne pas s'interroger sur les conséquences sur les apprentissages d'une trop forte centration sur la déficience de l'enfant. Ainsi, l'élève sourd, selon qu'il est considéré comme "déficient audio-vocal " ou "efficient visio-gestuel ", verra l'énergie et l'attention des personnes qui l'entourent se concentrer davantage sur la rééducation de son audition et de sa parole ou sur le développement de la communication gestuelle. L'inversion de perspective n'est pas indifférente si l'on admet que le regard porté sur l'enfant, dès sa naissance, participe de la construction de son identité. Mettons-nous à la place de l'enfant qui, dès le plus jeune âge, va vivre et construire au quotidien, à travers le regard de ses proches, le sentiment de n'être pas celui qu'il faut être. Né sourd, il assiste à la sidération, l'impuissance, la tristesse, voire la colère de ses parents au moment du diagnostic de surdité, très 
souvent il ne comprend pas les raisons de ce changement brutal, mais il va percevoir les modifications de son environnement. L'immense majorité des personnes qui l'entourent, entendantes pour la plupart, n'auront de cesse d'essayer de réduire sa déficience, de la compenser, voire pour certains de retrouver l'enfant entendant qu'ils viennent de perdre. II rencontrera de nombreux professionnels qui s'intéresseront à ses oreilles, puis rapidement lui demanderont d'écouter, d'entendre, enfin de parler, mesurant, valorisant le moindre progrès dans ce domaine. Afin d'atténuer la déficience constatée, des moyens considérables seront consacrés à la réhabilitation de son audition et de sa parole, lui donnant à voir que l'intérêt de l'autre à son égard réside essentiellement dans sa capacité à ne plus être ce qu'il est, à devenir plus entendant, plus parlant. L'enfant assiste aussi à la joie de sa famille dès qu'il prononce un son, un mot, aux félicitations lorsqu'il donne une bonne réponse et au désarroi, à la déception, voire à l'énervement quand il n'y parvient pas.

À l'école, dans un contexte oraliste, c'est-à-dire quand le choix de la communication audio-vocale est privilégié, on attend de lui qu'il réussisse à comprendre la parole vocale et à parler, il est fréquemment évalué sur capacité à " oraliser ". Rapidement il se construit autour de cet objectif, ultime récompense à tous ses efforts, au point parfois qu'il ne se considère lui-même pas comme sourd, refusant toute aide qui le renverrait à sa déficience.

Comme chacun, ce qu'il veut avant tout c'est être reconnu et valorisé, il veut réussir. Comment se construira celui qui ne parvient pas à entendre, ni à parler et qui n'est pas à la hauteur des espérances placées en lui?

Nous rencontrons régulièrement de nombreux enfants qui ne réussissent pas à entendre et à parler, simplement parce qu'ils sont sourds, l'aurait-on oublié ?

Paradoxalement, la déficience semble absorber une grande partie de l'énergie disponible, tant chez l'enfant lui-même que chez ses éducateurs et conduire finalement, parfois, à nier sa propre évidence. Tout se passe comme si l'enfant n'était pas sourd, puisqu'on lui demande à chaque instant d'entendre. Ce déni de surdité, mis en évidence par le sociologue Bernard Mottez, est fréquemment décrit par les adultes sourds. En tant que déficience de l'audition, la surdité est à double tranchant. Obnubilant l'entendant qui projette en elle, parfois jusqu'au refoulement, sa peur de perdre l'audition, elle est pourtant invisible et se fait oublier. Dans les deux cas l'expérience du déni est bien là.

Le deuxième écueil, sans doute lié au premier, par un effet de contrepoids, amène le balancier à une autre extrémité. Nous évoquions plus haut l'enfant sourd vu comme efficient visio-gestuel et non plus comme déficient auditif. Cette perspective porte effectivement en elle toutes les promesses d'une représentation non plus en creux mais en plein, avec ce que cela peut impliquer pour l'élève de confiance en soi, indispensable moteur interne des apprentissages. Elle consiste à envisager positivement la surdité comme une différence de potentialité. Or, depuis les années soixante-dix, cette revendication légitime à modifier dans ce sens le regard porté sur la surdité, et à réintroduire la langue des signes dans l'éducation des enfants sourds, conduit parfois à masquer certaines difficultés et à ne pas prendre en compte que, dans certaines situations, l'enfant sourd se trouve handicapé par le fait qu'il n'entend pas. En dépit de toutes les qualités qu'il possède par ailleurs, qui l'amèneront à 
apprendre selon des processus similaires à ceux des entendants, il est indéniable que l'enfant sourd n'a pas un accès au français aussi facile que l'enfant entendant, ce qui retarde son entrée dans l'écrit.

On peut comprendre ce qui a pu pousser certains à minimiser, voire à nier les conséquences de la surdité. Ce déplacement idéologique s'est construit en réaction contre les positions pédagogiques dominantes entre la fin du dix-neuvième siècle et la fin du vingtième, trop inspirées par une conception médicale de la surdité, fondée sur la recherche de la pathologie, la thérapeutique et son corollaire, la rééducation.

De façon générale, l'évolution des conceptions du handicap sous l'impulsion de I'OMS, visant à distinguer la déficience du handicap éventuel qu'elle génère et à envisager la personne dans sa globalité et non plus uniquement au regard de ses incapacités, a pu produire les mêmes effets de balancier, aboutissant parfois à gommer complètement l'idée du handicap, jugée trop stigmatisante. Voyons jusqu'où cela peut mener. Si l'enfant sourd est avant tout un enfant comme les autres, ayant des capacités visio-gestuelles indéniables et une intelligence comparable à celle des entendants, il suffirait pour les exploiter de lui proposer une voie d'accès aux connaissances visio-gestuelle, et donc comme langue de travail la LSF, c'est l'option adoptée par les projets bilingues. Le pas est vite franchi de ne pas tenir compte des implications de la surdité sur l'acquisition du français. On observe ainsi des dispositifs qui semblent ignorer que l'apprentissage de l'écrit, pour un élève n'ayant pas ou peu d'accès au français oral, ne pourra suivre la même démarche, ni la même progression, que celles adoptées pour les enfants entendants l'ayant entendu, acquis et pratiqué naturellement, pendant environ deux années avant leur entrée à l'école maternelle et environ cinq ans avant leur entrée en CP.

II faudra un dispositif qui permette à l'élève sourd de faire les expériences communicationnelles et langagières en français comme en LSF, auxquelles il aura, la plupart du temps, échappé dans son environnement familial, dans un cadre adapté, parfois incompatible avec le rythme d'une classe de trente élèves entendants.

Nous ne suggérons pas pour autant que l'élève sourd doive être scolarisé à part dans toutes les disciplines, ni même que ce soit un passage obligé pour tous les sourds, mais simplement qu'une évaluation fine des besoins de l'élève sourd en matière $d^{\prime}$ 'acquisition langagière peut conduire à concevoir un dispositif d'apprentissage langagier ponctuel ou plus régulier, n'omettant pas qu'il est sourd et qu'il a peut-être été privé d'expériences significatives dans ce domaine.

\section{DE LA PENSÉE MUETTE À « L'ESPRIT QUI ENTEND ॥: UN DIALOGUE DE SOURDS}

Les sourds ne peuvent que contredire l'idée de Boileau selon laquelle " ce qui se conçoit bien s'énonce clairement et les mots pour le dire arrivent aisément ". Dans une conception aristotélicienne simpliste des rapports entre pensée et langage, où la langue serait l'expression par excellence de la pensée, celle des sourds pourrait bien paraître muette et donc inexistante et il faut reconnaître que même une pensée exprimée dans une langue que l'on ne peut "com-prendre " est encore très souvent assimilée au néant. La sidération s'empare de l'observateur néophyte quand il prend connaissance de ce qui est exprimé par une personne sourde dès 


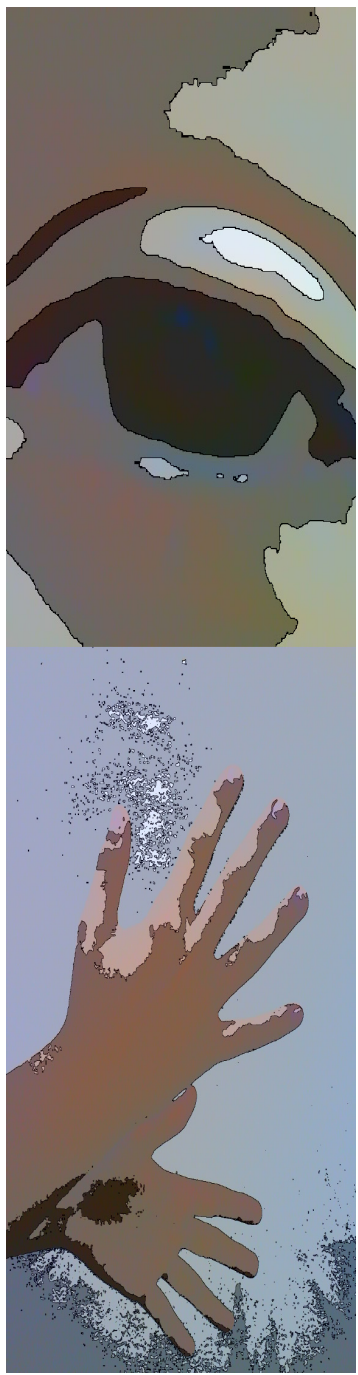

lors qu'un interprète lui prête voix. Celui qui paraissait moins intelligent et moins savant quand il s'exprimait dans un mauvais oral ou dans un geste incompris modifie sensiblement le regard porté sur lui dès lors que sa pensée devient appréhendable.

On constate souvent chez les élèves sourds que des capacités intellectuelles évidentes, manifestées lors d'activités non verbales se trouvent limitées dans leur expression par le fait qu'on leur demande de les verbaliser en français oral, ce que beaucoup d'entre eux ne parviennent pas à faire.

La communication étant un phénomène social, nécessairement partagé par au moins deux personnes, le handicap généré par la déficience d'un seul interlocuteur se trouve lui aussi partagé par les autres. Plusieurs réactions peuvent être observées de part et d'autre, débouchant parfois sur des comportements violents, voire une rupture de communication. Les interlocuteurs peuvent avoir tendance:

- à limiter leur inconfort en ignorant l'autre et à faire comme d'habitude,

- à parler de choses simples, de façon brève pour atténuer les difficultés: faire court, faire simple,

- à dire qu'ils ont compris ou ne pas dire qu'ils n'ont pas compris, pour mettre fin au malaise et inversement se persuader qu'ils ont été compris: faire semblant. Certains pourront même fuir la communication, se replier sur eux-mêmes ou devenir agressifs.

L'intercompréhension repose aussi sur la capacité des interlocuteurs à identifier et anticiper les incompréhensions.

L'illusion d'intercompréhension est fréquente dans toute communication, cela vaut encore davantage dans le cas d'une communication audio-vocale, si l'un des interlocuteurs est sourd.

La relation enseignant-élève va se trouver plus ou moins affectée selon le niveau de confort de l'un et de l'autre, il suffit qu'un seul des deux ne soit pas à l'aise pour cela affecte l'ensemble de la communication. Aussi, nous ne pouvons qu'insister sur le fait que, dès son entrée à l'école maternelle, on puisse réunir les conditions d'une communication confortable au sein de la classe.

La pensée et les mots se pliant plus aisément à la volonté de leur créateur que, dans les faits, les systèmes et les acteurs réels, la question paraît simple en théorie. II s'agirait de se livrer à un calcul de rentabilité, dans les domaines: affectif, cognitif, social, voire économique, faisant apparaître le rapport coût/bénéfice pour chacun des acteurs. L'art d'évaluer de façon prospective la capacité d'un dispositif à ménager, dans un confort équitable pour tous les interlocuteurs, des temps d'expérience et 
de communication suffisants pour permettre l'acquisition, dans les meilleurs délais, chez l'élève sourd, d'une langue de travail, sera déterminant, mais à dire vrai pas si simple en pratique. Deux options se profilent: une communication en langue française ou une communication bilingue, comme le stipule l'article L. 112-2-2. de la loi du 11 février 2005: "Dans l'éducation et le parcours scolaire des jeunes sourds, la liberté de choix entre une communication bilingue, langue des signes et langue française, et une communication en langue française est de droit. "

Plusieurs critères peuvent présider au choix de la langue d'apprentissage de l'élève.

Il importe que l'enfant ne subisse pas de pressions importantes de la part de la famille ou des professionnels, le poussant à parler ou à signer, ou, au contraire, à ne pas parler ou ne pas signer. De telles pressions risqueraient de générer des tensions internes difficiles à assumer. C'est pourquoi le comportement et les stratégies de l'enfant, qu'elles soient vocales ou gestuelles, doivent être observés, en situation de communication naturelle courante, afin de déterminer son mode privilégié. II n'y a pas de risque pour l'enfant à être exposé à une communication gestuelle, cela ne l'empêchera pas de communiquer vocalement s'il peut le faire. Par contre, le risque d'être privé de toute communication et d'entrer difficilement dans le langage et les apprentissages est grand, si l'enfant n'accédant pas à la communication vocale n'a pas été " autorisé " à adopter une communication gestuelle.

Trois indicateurs devraient permettre à l'observation de s'orienter vers l'un ou l'autre choix, appartenant en dernier ressort à l'enfant sourd lui-même:

- le confort: la langue de communication et d'apprentissage doit être économique pour l'élève, faute de quoi elle ne se construira que très imparfaitement et ne remplira pas toutes ses promesses,

- la précocité: la langue choisie pour les échanges et les apprentissages doit pouvoir être acquise rapidement,

- la richesse: le choix fait par l'environnement éducatif doit aboutir à l'acquisition d'une langue riche. Quelques mots ou quelques signes ne suffiront pas aux apprentissages ultérieurs: scolaires, culturels et sociaux. II va de soi que des apprentissages aussi complexes que la lecture de textes ou la résolution de problèmes ne pourront se satisfaire d'une demi-mesure, faute de quoi on risquerait bien de retarder les apprentissages, ce qui constituerait un handicap supplémentaire.

Si l'on peut se contenter, dans des temps courts, d'une communication partielle, où les efforts à fournir sont importants de part et d'autre, c'est ce qui ne manquera pas d'arriver souvent aux personnes sourdes tout au long de leur vie, en revanche, le moment de la découverte et de l'apprentissage nécessite une bonne intercompréhension et une langue de travail suffisamment maîtrisée pour que l'élève et le professeur ou les parents ne consacrent pas toute leur énergie à essayer de se comprendre.

\section{SOURDS ENSEMBLE, PARMI LES ENTENDANTS, POUR QUEL PARTAGE?}

Considérant que, parmi les missions fondamentales de l'école maternelle, la socialisation et la maîtrise de la langue apparaissent comme prioritaires, la difficulté tant théorique que pratique de la réflexion autour des choix de dispositifs de scolarisation et d'apprentissage pour les élèves sourds tient à l'étroite marge manœuvre dont 
on dispose, compte tenu des risques évoqués plus haut. La pertinence des choix faits à l'école maternelle se révélera particulièrement déterminante.

Quatre grands types de données doivent être croisés et mis en synergie:

- Les ressources, faiblesses et contraintes personnelles de l'enfant et de sa famille.

- Celles de l'établissement, de la classe d'accueil et de l'enseignant de l'enfant.

- Celles du contexte institutionnel et réglementaire national ou local.

Comment acquérir une langue qui puisse être un levier pour tous les apprentissages, dans un espace et un temps adaptés, tout en apprenant à vivre ensemble, et donc avec les entendants?

Dans quelle mesure et jusqu'où le dispositif commun d'apprentissage des élèves entendants peut-il prendre en compte les besoins particuliers de tel ou tel élève sourd?

Si le mode de communication privilégié de l'enfant est l'oral et qu'il est déjà développé d'une façon comparable à celui des entendants du même âge (le plus souvent chez les enfants les moins sourds ou ayant déjà tiré un large profit de moyens techniques de compensation, tels que les appareils de correction auditive, techniques de rééducation ou aides à la communication vocale), on peut envisager une scolarisation dans une classe d'entendants dont la langue de communication sera le français, moyennant certains aménagements acoustiques, matériels et quelques adaptations pédagogiques. Des moments consacrés à la communication orale, en individuel ou en groupe restreint, peuvent être nécessaires.

Si le mode de communication est la LSF (souvent chez les élèves sourds de parents sourds, ou entendants ayant fait le choix de l'éducation bilingue avant la maternelle) ou si une communication gestuelle émergente s'est élaborée au sein d'un environnement non signeur, accueillant favorablement la gestualité de l'enfant, l'inclusion dans les dispositifs communs de scolarisation est possible, dans tout ou partie des situations d'apprentissage, moyennant la présence au sein de ces dispositifs, d'enseignants compétents en LSF : des situations de co-enseignement avec un enseignant sourd ou entendant maîtrisant la LSF sont alors proposées. Dans certaines activités, mobilisant peu la langue, les élèves sourds pourront être pris en charge dans la classe uniquement par l'enseignant d'accueil (il est alors utile qu'il soit initié à la LSF) ou accompagné par une auxiliaire de vie scolaire maîtrisant la LSF.

Dans le cadre des parcours bilingues (LSF/français) décrits par l'arrêté du 15 juillet 2008 (BOEN, $n^{\circ} 33,4$ septembre 2008), fixant le programme de l'enseignement de la Langue des signes française à l'école primaire, des regroupements d'élèves sourds peuvent être nécessaires pour l'acquisition et amélioration des deux langues dans des dispositifs d'apprentissage adaptés au développement langagier de l'enfant.

Si l'enfant ne semble pas avoir de moyen de communication à son entrée à l'école maternelle, il apparaît urgent de l'exposer à la LSF, s'il ne l'a jamais été, afin de ne pas compromettre ses chances d'apprentissage. Si le choix de la famille est oraliste, ceci ne signifie pas nécessairement qu'on renonce au projet d'acquisition de la langue orale, mais simplement qu'on tente de pallier rapidement l'absence de communication qui pourrait être préjudiciable au développement psychologique et intellectuel de l'enfant. 
Quel que soit le mode de communication et de scolarisation de l'élève, il importe qu'il ne soit pas isolé et puisse rencontrer d'autres enfants sourds comme lui ainsi que des adultes sourds, se construire en tant que sourd et se projeter dans l'avenir en tant que tel. À l'école maternelle, plus qu'ailleurs, un mode de communication direct doit être privilégié, car l'enfant est trop jeune pour comprendre une communication triangulaire médiatisée par un interprète français/LSF ou un codeur en LPC. Aussi, la présence d'autres locuteurs de la LSF ou d'élèves sourds ou malentendants comme lui présentant un profil langagier comparable est primordiale, afin qu'il dispose d'espaces de communication plus confortables que les grands groupes d'entendants dont les interactions adoptent un rythme souvent trop rapide pour qu'il s'y insère en toute confiance.

\section{LA CONOUÊTE DE L'ESPACE SONORE: UNE CHIMĖRE?}

Alors que pendant près de cent ans on a invalidé le potentiel éducatif et pédagogique de la LSF, en la laissant aux portes des institutions, au profit exclusif de l'oralisme, on pourrait bien céder, ça et là, à la tentation inverse et faire du son une incongruité dans l'éducation des sourds. Les recherches et observations menées par l'INS HEA montrent que le son, quelle qu'en soit l'origine: organique, mécanique, musical, vocal ou linguistique..., est un objet d'apprentissage indispensable pour les élèves sourds ou malentendants, qui plus est, est un centre d'intérêt inépuisable pour eux, sans doute parce qu'il symbolise le lien qu'ils entretiennent avec nous, les entendants. II se peut que cet apprentissage se limite chez eux à une représentation partielle ancrée dans des expériences plus visuelles et vibratoires que véritablement sonores, qu'importe, c'est le cerveau qui entend et comprend, il peut bien se passer de l'oreille qui ne fait que percevoir et transmettre le son. La difficulté tient à nouveau à notre capacité d'enseignants à aller jusqu'où l'on peut aller, sans aller au-delà du possible, ni au-delà du souhaitable.

On a vu combien une trop grande centration sur l'oreille et la voix invalides risquait de devenir contre productive, il s'agit bien au contraire ici de s'appuyer sur les potentialités poly-sensorielles et intellectuelles mobilisables, sans se donner pour objectif de développer des performances, mais de construire des apprentissages progressifs et raisonnés, raisonnables surtout.

Profitant de l'appétence naturelle des jeunes enfants sourds ou entendants à explorer, observer, jouer avec les objets, avec leur corps et leur voix, les expériences quotidiennes vécues à l'école maternelle doivent devenir autant d'occasions de découvrir que tel mouvement produit du bruit, que tel autre n'en produit pas. La langue qui servira à commenter, caractériser, catégoriser les classes d'objets, sonores ou non, qu'elle soit le français ou la LSF, permettra de nommer, de comparer, de définir les sons. Certaines caractéristiques d'une séquence de parole ou de musique peuvent être appréhendées par les élèves sourds, notamment toutes celles qui peuvent être perçues visuellement, par le mouvement ou par la vibration, ou conçues intellectuellement: c'est le cas du rythme et de l'intensité. La hauteur des sons qui constitue la mélodie ou l'intonation semble plus directement liée à l'audition, mais peut être visualisée par des mouvements et explicitées par la parole. 
Des activités ponctuelles spécifiques peuvent être conduites avec l'enfant sourd, visant l'écoute, la production sonore ou musicale, à condition qu'elles ne mobilisent que les capacités qu'il a et, si elles ont un sens pour lui, un usage mécanique des sons de la langue ou de la musique, dénué de sens, d'intention de désir est à proscrire. Afin de ne pas empiéter sur le registre de la rééducation de la parole et de l'audition, qui ne relève pas de son champ d'intervention, l'enseignant devra s'en tenir à la construction de savoirs et de savoir-faire appuyés sur une évaluation des compétences acquises de l'élève. L'apport que peut constituer pour l'enseignant la lecture des audiogrammes ou celle des bilans orthophoniques à cet égard, afin de ne pas aller au-delà du possible, peut être précieux, mais l'évaluation sommative des potentialités auditives ou articulatoires de l'élève n'a pas lieu d'être à l'école, car c'est ce que fait l'élève, ce qu'il veut faire et sait faire qui sert de fondement aux apprentissages ultérieurs.

Les recherches-actions menées à I'INS HEA tant dans le champ de la musique que dans celui de l'apprentissage de la LSF et du français, font apparaître une réelle envie des élèves sourds d'entrer dans le monde entendant et de le comprendre. L'école maternelle est souvent pour eux le lieu de la découverte de leur différence et de la construction progressive de leur identité de sourd, les activités de découverte et de compréhension du sonore sont à ce titre primordiales. En fonction des capacités auditives de l'enfant, de son niveau de langue et de son bagage expérientiel dans ce domaine, ces activités pourront être conduites ou non avec les élèves entendants. Pour certains élèves elles nécessiteront des adaptations telles qu'elles devront être menées séparément. Des regroupements d'élèves sourds ou malentendants ponctuels ou plus réguliers peuvent être envisagés.

Les processus d'apprentissage des élèves sourds, tout en empruntant des voies différentes, sont de même nature chez les élèves sourds et entendants, ils sont gérés par les mêmes structures, selon les mêmes déterminations. Ainsi, l'expérience, le langage et la conceptualisation entretiennent les mêmes rapports étroits et se renforcent mutuellement chez l'élève entendant comme chez l'élève sourd. Pour que ce dernier puisse établir les liens entre les objets du réel, les objets de pensée et les objets de langage, nécessaires à la compréhension du monde et à la communication avec les autres, il lui faut, dès le plus jeune âge, une langue confortable pour décrire, nommer, penser et communiquer, qu'elle soit le français ou la LSF. La langue a elle seule ne suffit pas il lui faudra également un bagage d'expériences variées et répétées: sensori-motrices, affectives ou relationnelles, accompagnées de langage dit " en situation ", et un bagage d'expériences communicationnelles et langagières " hors situation ", l'amenant vers le langage d'évocation. En cela l'élève sourd ne se distingue de l'élève entendant que par les conditions et modes d'accès aux apprentissages. C'est ce qui peut justifier qu'il doive dans certains cas bénéficier de dispositifs qui lui sont dédiés.

II est finalement assez rassurant de voir que les situations les plus atypiques, où le pathologique, l'anormal, voire la monstruosité nous fascinent, mais aussi nous font nous réfugier parfois dans des positions de repli défensif, permettent souvent de penser l'ordinaire avec plus d'acuité que les stéréotypes. La malvoyance met en avant de façon particulièrement saillante la problématique de la lisibilité, le handicap 
moteur celle de l'accessibilité aux locaux et la surdité cristallise les questions liées à la communication. Ce sont au fond des phénomènes, des structures et des processus banalement humains, parfois impensés, qui poussent chacun à se décentrer pour voir et accepter l'impensable.

\section{Bilbiographie}

Bertin (F.), AbDAllah-PRETCEILle (M.), " Les enjeux d'une éducation bilingue et biculturelle pour les élèves sourds ", La nouvelle revue de l'adaptation et de I'intégration scolaire, n²3, Cnefei, Suresnes, 2003.

BRUNER (J.), Comment les enfants apprennent à parler, Forum Éducation Culture, Retz, 2002.

COURTIN (C.), "Le développement de la conceptualisation chez l'enfant sourd; synthèse des travaux existants ", La nouvelle revue de l'adaptation et de l'intégration scolaire, n 17, Cnefei, Suresnes, 2002.

CUXAC (C.), "La Langue des signes française; les voies de l'iconicité ", Faits de Langues, Ophrys, Paris, 2000.

FUSELLIER-SOUZA (I.), Sémiogenèse des langues des signes. Étude de langues des signes primaires pratiquées par des sourds brésiliens, Paris VIII.

FUSELLIER-SOUZA (I.), "La création gestuelle des individus sourds isolés ", Acquisition et interaction en langue étrangère, [en ligne] 2005, disponible sur: http://aile.revues.org/document537.html

GOLDIN-MEADOW (S.), "When does gesture become language? A study of gesture used as a primary communication system by deaf children of hearing parents ", in GIBSON \& INGOLD, Tools, Language and Cognition in Human Evolution, Cambridge University Press.

MOTTEZ (B.), Les sourds existent-ils? L'Harmattan.

VANBRUGGHE (A.), " Apprendre à lire sans entendre, apprendre à lire sans parler: controverses et tabous ", La nouvelle revue de l'adaptation et de l'intégration scolaire, $n^{\circ}$ spécial, Cnefei, Suresnes, juin 2005.

YAU (S.-C.), Création gestuelle et début du langage. Création de langues gestuelles chez les sourds isolés. Langages Croisés, Hong-Kong. 